\title{
P I 9-46. Co-delivery of mucosal chemokine plasmids in a systemically delivered DNA vaccine elicits systemic and mucosal immune responses in mice and macaques
}

\author{
KA Kraynyak*1, MA Kutzler², B Pahar ${ }^{3}$, A Sylvester ${ }^{2}$, J Yan¹, D Carnathan ${ }^{1}$, \\ AS Khan ${ }^{4}$, N Sardesai ${ }^{4}$, Z Moldoveanu ${ }^{5}$, J Mestecky ${ }^{5}$, MR Betts ${ }^{1}$, P Marx ${ }^{3}$ and \\ DB Weiner ${ }^{1}$
}

Address: ${ }^{1}$ Department of Pathology and Laboratory Medicine, University of Pennsylvania, Philadelphia, PA, USA, ${ }^{2}$ Drexel University College of Medicine, Philadelphia, PA, USA, ${ }^{3}$ Tulane National Primate Research Center, Covington, LA, USA, ${ }^{4}$ VGX Pharmaceuticals, The Woodlands, USA and ${ }^{5}$ University of Alabama at Birmingham, Birmingham, AL USA

* Corresponding author

from AIDS Vaccine 2009

Paris, France. 19-22 October 2009

Published: 22 October 2009

Retrovirology 2009, 6(Suppl 3):P366 doi:10.1 186/1742-4690-6-S3-P366

This abstract is available from: http://www.retrovirology.com/content/6/S3/P366

(C) 2009 Kraynyak et al; licensee BioMed Central Ltd.

\section{Background}

The induction of mucosal immunity is a crucial goal for HIV vaccines. DNA vaccines which are non-live/non-proliferating have had limited success in this area. Mucosal immune cell homing is in part controlled by a subset of chemokines (CCL27, CCL28 and CCL25). We hypothesized that a DNA vaccine encoding antigen and chemokine could induce mucosal immunity.

\section{Methods}

Mice were systemically co-immunized with pHIV-1gag/ pol and chemokine adjuvants. Rhesus macaques $(n=5 /$ group) were immunized intramuscularly with antigenic plasmids plus/minus each chemokine. For rhesus optimized CCL27, CCL25, and CCL28 plasmids, optimized/ consensus macaque pol and sooty mangabey consensus gag/env plasmids were generated.

\section{Results}

Mice: Co-immunization with chemokines induced significant enhancement of HIV-1 specific CD8+ T cell IFNgamma secretion in the periphery and TNF-alpha, IL-2 and IFN-gamma by gut lymphocytes. Co-immunization with mucosal chemokines augmented HIV-1-specific sIgA in sera/fecal samples. Similar immunogenicity data was observed to Influenza A/PR8/34 hemagglutanin plasmid including responses that neutralized virus and protected mice from morbidity/mortality associated with lethal mucosal challenge. Macaque: In the periphery, we observed significant IFN-gamma in all groups $(\sim 6,000$ SFU each). However intracellular cytokine staining on mucosal lymphocytes showed a trend toward an increase in CD8+T cells secreting TNF and IL-2 in CCL27 COimmunized macaques, levels greater than those observed in infected animals. Enhanced antigen-specific IgA was also detected in sera of chemokine-vaccinated macaques. A mucosal challenge is scheduled to determine if the functionality and phenotype of vaccine-induced immunity, either at the mucosa or periphery, is a driving determinant of protection.

\section{Conclusion}

The results of this study will be critical to the development of an effective vaccine against HIV. This is the first example of the use of mucosal chemokines to influence a DNA vaccine strategy, suggesting a novel approach for manipulation of vaccine-induced immune responses. This work is supported by NIH funding (HIVRAD). 